\title{
Nécropoles Préhistoriques
}

Colette Roubet et Slimane Hachi

\section{OpenEdition}

Journals

Édition électronique

URL : https://journals.openedition.org/encyclopedieberbere/2692

DOI : 10.4000/encyclopedieberbere.2692

ISSN : 2262-7197

\section{Éditeur}

Peeters Publishers

\section{Édition imprimée}

Date de publication : 4 octobre 2012

Pagination : $5320-5338$

ISBN : 978-90-429-2640-0

ISSN : 1015-7344

\section{Référence électronique}

Colette Roubet et Slimane Hachi, « Nécropoles Préhistoriques », Encyclopédie berbère [En ligne], 33 | 2012, document N22, mis en ligne le 23 novembre 2020, consulté le 17 février 2022. URL : http:// journals.openedition.org/encyclopedieberbere/2692 ; DOI : https://doi.org/10.4000/ encyclopedieberbere.2692

Ce document a été généré automatiquement le 17 février 2022.

(c) Tous droits réservés 


\title{
Nécropoles Préhistoriques
}

\author{
Colette Roubet et Slimane Hachi
}

1 Cinq importantes nécropoles préhistoriques sont connues en Afrique du Nord. Il s'agit de Taforalt* (Maroc oriental), d'Afalou Bou-Rhummel* (Babors, Algérie), de Columnata* (Tiaret, Algérie) d'El-Kiffen et Skhirat (Maroc atlantique). Sur le territoire septentrional ces nécropoles occupent des lieux choisis, privilégiés, modifiés, utilisés entre 22000 et 10000 ans pour les deux premières et entre 7000 et $3000 \mathrm{cal} \mathrm{BC}$, pour les suivantes. Divers travaux thématiquement cloisonnés leur ont été consacrés. Aucune étude d'ensemble n'a abordé le thème comportemental. En effet, durant le $\mathrm{XX}^{\mathrm{e}}$ siècle les rapports de fouilles faisant état d'inhumations, utilisèrent un langage neutre, ne rapportant que des faits matériels bruts. Certains auteurs s'attachèrent à préciser la position des squelettes dans des sépultures individuelles ou collectives, celle de documents alentour, d'agencements ou de structures présentes. Toutes les informations relevées ont utilisé une terminologie objective, descriptive, rendant compte d'un état physique osseux, sans évocation de l'être humain vivant, social, sans suggestion d'une perception de rupture dans le corps social, ni de mention sur l'accompagnement de la communauté. Pourquoi? - Sans doute par crainte de glisser subjectivement vers des comportements actuels, supposés faire perdre la maîtrise de ce sujet du passé, mais surtout par manque de vision anthropologique, d'outils de langage et de questionnements en adéquation avec une documentation délibérément perçue comme réduite à cette ultime apparence matérielle. L'adoption d'outils d'une interrogation adaptée à l'anthropologie sociale pourrait faire resurgir d'infimes traces d'un sens profond du fait funéraire préhistorique. Si pertinentes et indispensables que soient les données strictement biologiques et archéologiques, sans doute s'inscriraientelles mieux encore dans un contexte comportemental et sociétal local et régional. Pouvons-nous atteindre ce palier de connaissances remontant à 22000 ? Certainement, en lançant un pont dans cette direction, sans marginaliser la durée écoulée jusqu'à nous pour retrouver en cet Homme les racines d'une permanence comportementale. En recherchant avec d'autres instruments aussi des bribes de préoccupations symboliques en relation avec ce fait fondamental qui relie tous les Sapiens les uns aux autres, tel est en effet l'objectif que s'est fixé S. Hachi (en préparation) pour faire surgir de la nécropole d'Afalou Bou-Rhummel, une immatérielle substance encore présente. 


\section{Deux nécropoles telliennes de l'ibéromaurusien*}

\section{La nécropole de la grotte des Pigeons à Taforalt* (Maroc)}

$2 \mathrm{Au}$ Maroc oriental la grotte des Pigeons près du village de Taforalt se situe dans le massif boisé des Beni Snassen, à $55 \mathrm{~km}$ au nord-ouest de la ville d'Oujda, à $500 \mathrm{~m}$ à vol d'oiseau du village de factuelle Tafoughalt. Orientée à l'Est, son ouverture dans la falaise calcaire est d'env. $30 \mathrm{~m}$, sa profondeur de $28 \mathrm{~m}$, elle domine la vallée du torrent Zegzel. «Signalée dès 1908 par le Dr Pinchon elle fut tardivement fouillée. De 1944 à 1947 Ruhlmann y effectua deux sondages profonds» (Roche 1953, p. 164). Cinq campagnes conduites (1951 et 1955) par l'Abbé Jean Roche ont fait connaître son contenu sans atteindre le substratum naturel. Elle conserve bien d'autres dépôts en place.

3 Le remplissage de la grotte comprend 12 niveaux (A-H), contenus dans plus de $8 \mathrm{~m}$ d'épaisseur, renfermant deux ensembles colorimétriques et culturels distincts. La stratigraphie conserve dans l'ensemble profond argileux et jaune une succession Atérien-Moustérien (niveau 11)-Atérien, correspondant aux niveaux H-D (désignés entre 12 à 8 ). Cet ensemble est surmonté par des blocs (niveau 6) sur lesquels s'élève l'ensemble supérieur grisâtre-noir, conservant l'Ibéromaurusien et ses sépultures entre E et $\mathrm{A}$ (niveaux 5 à 1).

D'autres travaux géologiques, sédimentologiques et micromorphologiques ont été effectués par Courty et al 1989, Courty et Vallverdu 2001 et Woodward et Golberg 2001.

La reprise de recherches en 2003 par une équipe Maroco-Britannique (Bouzouggar et al. 2008 ; Barton et al. 2007, 2011; Taylor et al. 2011) établit, avec l'appui de récentes données chronologiques, l'état actuel des dépôts. Retrouvant les deux ensembles décrits ci-dessus, ces auteurs observent que les premières installations ibéromaurusiennes commencent, - dans les secteurs explorés -, avec la fin des dépôts jaunes: "The upper layers of the yellow series in sector 8 also contain evidence for iberomaurusian occupation with the oldest date obtained for this period being $17515 \pm 75$ (OXA-16273, Barton et al 2007)» (Taylor et al. 2011, p. 2). La séquence chronostratigraphique situe les dépôts gris entre 15 057-14 201 cal BP et 12 942-12 649 cal BP (Cf. leur Tableau 1). Nous rapprocherons plus loin ces repères de ceux obtenus à Afalou.

6 D'autre part, si la majeure partie des témoins anthropologiques découverts par J. Roche provient de la série grise (nécropoles I et II non localisée dans les publications de J. Roche, ni sur les plans de N. Barton), les investigations nouvelles révèlent l'existence dans le secteur 10 de nouvelles sépultures «work on iberomaurusian burials in sector 10, includes assessment of the health and nutritional behaviour and mortuary practices of the population of Taforalt.. » (Taylor and al. 2011, p. 4).

7 Ces informations récentes donnent aux anciens travaux un cadre paléoenvironnemental et chronologique dans lequel situer les 183 individus anthropologiquement identifiés. Tous sont des Africains du type de Mechta-Afalou.

Comment J. Roche signale-t-il ses découvertes anthropologiques et les sépultures ? Ses propos prennent ici une valeur nouvelle et forte :

«Au cours de la campagne de 1951, le niveau A avait fourni des fragments humains appartenant à au moins deux individus, dont le crâne d'un sujet masculin ayant subi une avulsion dentaire; le niveau D un petit fragment de voûte crânienne, 
malheureusement très incomplet. Au printemps 1952, l'effritement des coupes formées de cendres extrêmement pulvérulentes permit d'entrevoir deux squelettes assez complets dans le niveau B. Il fut décidé de fouiller cette partie de la grotte. Le niveau A permit de découvrir un véritable ossuaire ibéromaurusien. Les restes plus ou moins complets d'une dizaine d'individus furent exhumés. La plupart des corps étaient couchés sur le dos, la tête tournée à l'Ouest, le visage tourné vers le soleil levant. Cette disposition avait déjà été remarquée par Barbin à la Mouillah (1912). Dans un cas, le rite funéraire semble certain : plusieurs corps de jeunes enfants avaient été déposés dans une fosse trapézoïdale, limitée sur trois côtés par des blocs de pierre. Contre le bloc nord, un corps de bébé était pelotonné, couché sur le côté gauche, la face tournée vers l'extérieur. Au centre, plusieurs corps d'enfants étaient allongés dans l'axe Est-Ouest. Sur leurs poitrines on avait déposé en étoile trois massacres de mouflons, maintenus en place par une pierre centrale, les cornes tournées vers l'extérieur » (Roche 1953, p. 379).

Revenons à ce niveau $\mathrm{A}$ et à sa présentation trop succincte; après le niveau 1 , sol superficiel de $0,10 \mathrm{~m}$ d'épaisseur, succède, dit-il, le niveau $\mathrm{n}^{\circ} 2$ qui apparaît comme un «sol cendreux riche en fragments de coquilles d'escargots, en restes osseux, pierres carbonisées et traces de foyers. 0,70 m à 1,85 m (niveau A) (Roche 1953, p. 375).

Ces données premières n'ont jamais été développées ni reprises dans les publications postérieures de l'auteur (Roche 1963), ni dans celles d'autres collaborateurs.

11 L'inventaire des individus anthropologiques (Férembach et al. 1962) fait état de 28 sépultures pour un total de 170 sujets seulement sur 183. Leur répartition révèle l'inhumation de 100 adultes et 70 enfants. Parmi les adultes : 39 sont masculins, 31 sont féminins et 10 sont adolescents. Parmi les enfants classés selon l'âge estimé au décès, les nouveaux nés jusqu'à 1 an sont au nombre de 45 , les autres décédés avant 2 ans sont 24 au total, puis 14 enfants ont un âge compris entre 2 et 5 ans, et l'âge de 17 jeunes et adolescents se situe entre 5 et 16 ans. On aura constaté la forte mortalité infantile (problème dû au sevrage) et périnatale en particulier (c'est-à-dire de la naissance jusqu'à un mois) dont D. Férembach (ibid. p. 17) pense qu'elle serait d'origine génétique, en relation avec un coefficient élevé de consanguinité (malformation du sacrum ou spina bifida), entretenue par une forte endogamie vraisemblable.

12 Une certaine surmortalité féminine entre 15 et 40 ans a aussi été relevée (Férembach ibid. p. 18) ; l'âge du décès des adultes avant 40 ans était fréquent et se situait plutôt vers 35 ans. Selon diverses procédures (suture, dentition, etc.) préconisées pour faire cette détermination, un seul individu, d'environ 47 ans, serait plus âgé. Nous n'exposerons pas les données anthropologiques et paléopathologiques du type de Mechta-Afalou déjà présentées par M.-C. Chamla et le $\mathrm{D}^{\mathrm{r}} \mathrm{J}$. Dastugue (52 squelettes pathologiques), ni celles d'Afalou retenues notamment dans l'EB (fasc. II, p. 184-192).

Rappelons que le terme de "nécropole » a d'abord été appliqué par J. Roche à la grotte de Pigeons de Taforalt, dès 1953, et qu'il servit en 1962 au titre de l'étude générale des squelettes humains qu'en fit D. Férembach, sans jamais atteindre la substance symbolique, si caractéristique du lieu. Aussi nous retournerons-nous vers ces quelques phrases citées ci-dessus pour souligner que c'est J. Roche qui, reconnaissant en fouille des inhumations spéciales, les examina avec attention. L'une d'elles fut analysée sans indifférence : il s'agit de celle réservée aux corps de plusieurs enfants, disposés dans une fosse, délimitée par des parements rocheux latéraux. J Roche nota le caractère d'offrande agencée, donné aux cornages et aux crânes de mouflons, posés " en étoile ", dit-il, maintenus par une pierre sur la poitrine des enfants, occupant le centre de cette structure. Partant de cette observation, on ne peut s'empêcher de rapprocher ces faits 
précis des activités de chasses spécialisées aux mouflons des adultes Ibéromaurusiens que nous connaissons mieux maintenant et qui se pratiquèrent à Taforalt. Un lien étroit s'exprimerait-il ici entre les forêts giboyeuses, les chasses organisées et le décès de tous ces enfants (véritable hécatombe, bien que le nombre en reste indéterminé) que la communauté pouvait avoir déjà considérés comme de futurs adultes chasseurs?

Autre observation de valeur, la position de la tête à l'Ouest, le visage tourné vers la vie et la lumière du dehors, notée pour les individus de l'ossuaire, et celle aussi du corps allongé sur le dos de plusieurs adultes inhumés, sans délimitation de leur espace. On sait que la position allongée fut réservée à certains individus et non donnée à tous, dans la nécropole d'Afalou Bou-Rhummel.

Rappelons enfin deux cas assez émouvants du traitement thérapeutique particulier donné par de réels spécialistes en soins et du cortège d'attentions quotidiennes qu'il nous faut implicitement supposer avec J. Dastugue parce qu'elles furent apportées par les membres d'une communauté contrainte à la sédentarité. Ces cas sont ceux que connurent deux individus adultes que le paléopathologiste J. Dastugue découvre et signale en chirurgien averti, transférant sur ces habiles prédécesseurs sans bistouri, ni équipement ad hoc, son admiration confraternelle de spécialiste moderne, passant dans l'esprit de son maître L. Manouvrier de la chirurgie classique et moderne à la chirurgie préhistorique (Dastugue 1959). Le premier cas est celui du squelette de la femme $\mathrm{n}^{\circ} \mathrm{XVIII}$ :

«Ayant subi un accident grave, atteinte d'une fracture de la clavicule et des deux avant-bras (au minimum), cette femme a pu survivre assez longtemps à ses blessures pour consolider ses fractures et ensuite développer une arthrose cervicale... De plus, si l'on en juge par l'aspect des cals et par celui de la tête humérale gauche, elle a dû être affectée d'une impotence fonctionnelle définitive à peu près complète de son membre supérieur gauche et partiellement droit. La survie prolongée de cette blessée grave suppose donc non seulement qu'on ne l'a pas supprimée, comme bouche inutile, mais encore qu'on l'a soignée et assistée pendant longtemps. Cela implique des notions de solidarité tribale déjà bien développée...»

Le second exemple concerne la trépanation de deux crânes (Dastugue 1962, p.158) :

«Pour moi le fait est établi sans contestation possible et il témoigne de deux choses. Tout d'abord la trépanation crânienne était connue et pratiquée antérieurement aux temps dits "Néolithiques". Certes nous ne sommes en présence que des premiers balbutiements de la technique et loin des audaces qui se feront jour par la suite. Pourtant le fait est là : les hommes de Taforalt savaient ouvrir un crâne au lieu d'élection et, avec assez d'habileté pour que le sujet survive et répare les bords de l'orifice osseux [...]. En second lieu, ce fait témoigne [...] de la mise au point de la technique mais encore de l'invention même de l'ouverture crânienne, qui supposent une réflexion déjà très élaborée. On peut évidemment discuter [...] sur les raisons premières qui ont conduit les Préhistoriques à cette pratique. Mais qu'elles soient d'ordre magique ou thérapeutique, elles demandent que l'homme qui les conçoit se soit notablement élevé au dessus des préoccupations purement végétatives. Elles supposent également une certaine organisation sociale puisque la réalisation de l'opération nécessite l'existence d'un opérateur et d'un patient liés par des rapports de consentement ou de subordination ».

Précisons que la trépanation a aussi été signalée à Skhirat (cf. infra). Ces propositions comportementales suggèrent tant de remarques qu'il faudra renouer avec les documents, réexaminer le contexte chirurgical et anatomique, mais aussi revenir aux sédiments qui conservent dans cette grotte privilégiée, plus qu'un sanctuaire, un 
«laboratoire thérapeutique avec ses instruments» plongés tous deux dans une atmosphère spéciale, et une substance symbolique évocatrice de l'interdépendance des activités du quotidien et du fait funéraire. Au Maroc, on ne retrouve cette complexité que dans le massif tutélaire de Beni Snassen.

\section{La nécropole d'Afalou Bou-Rhummel des Babors (Algérie orientale)}

Ainsi que cela a été mentionné ci-dessus, l'EB (fasc. II, p. 182-193) a présenté diverses informations sur le site littoral et les découvertes pionnières qui seront introduites et articulées à celles que fournissent les derniers travaux approfondis (Hachi 1995, 1996, 1999, 2003a, 2003b, 2006 ; Hachi et al. 2002 ; Roubet et Hachi 2000).

Le gisement d'Afalou Bou-Rhummel conserve toujours, dans le vaste abri sous roche non vidé de son contenu - la première nécropole préhistorique découverte en Afrique du Nord par C. Arambourg en 1927 (Aramboug et al 1934). D'une cinquantaine de sujets mise au jour, on a individualisé 26 hommes, 14 femmes et 10 enfants. Dix autres sujets sont ensuite apparus lors des travaux de S. Hachi.

Dans les quatre niveaux stratigraphiques reconnus en 1927 par C. Arambourg, c'est le premier, d'environ quatre mètres d'épaisseur grisâtre, qui préserva 48 sujets (têtes osseuses) à l'intérieur d'une épaisseur sédimentaire d'à peine $70 \mathrm{~cm}$, comprise entre $3,25 \mathrm{~m}$ et $4 \mathrm{~m}$. Six individus à peu près complets se trouvaient répartis sur une aire de 4 x $3 \mathrm{~m}$ environ, allant du fond de l'abri jusqu'à l'aplomb de la voûte. Cependant, l'absence d'information concernant les 42 autres individus, trouvés pêle-mêle a conduit les auteurs postérieurs à admettre, comme C. Arambourg, l'idée d'un réel amoncellement indescriptible, " un charnier ». Pourtant cela n'a pas été formellement établi. On peut même envisager que de minutieuses observations d'anthropologie physique auraient pu permettre de reconnaître et individualiser certains d'entre eux, au moins. Quoiqu'il en soit C. Arambourg qualifia cet ensemble d'« ossuaire» et le décrivit comme « une masse de contour à peu près circulaire dont le centre correspond exactement à la projection horizontale de la voûte. C'est un véritable entassement et on peut se demander s'il n'existe pas une relation entre la situation de ce charnier et la cheminée de la voûte qui aurait pu servir à la précipitation des corps par cette voie » (Ibid. p. 22).

21 Dans ce que S. Hachi désigne aujourd'hui comme devant être le niveau III de la stratigraphie de C. Arambourg, deux autres inhumations furent mises au jour en 1927, dans un sédiment jaunâtre à rouge, d'un mètre d'épaisseur, reposant à $5,75 \mathrm{~m}$ de profondeur, sur un niveau argileux, non numéroté par le fouilleur, qu'il aurait fallu individualiser et qui représente désormais le niveau II. Le squelette complet d'un sujet masculin adulte $\mathrm{H} 28$ et le crâne d'un enfant $\mathrm{H} 16$ se sont trouvés associés ; la tête de l'enfant posée sur le pied droit de l'adulte, la face tournée vers le sol. L'adulte $\mathrm{H} 28$, à la dolichocéphalie accentuée, avait été inhumé en décubitus dorsal allongé, la tête tournée vers l'ouverture de l'abri, lui aussi, comme à Taforalt, les bras allongés, la main droite ramenée vers le pubis. Sous son menton se trouvaient quelques galets de silex non taillés et, au sommet du crâne, un dépôt de fer oligiste de plus d'un kilo. Au milieu de ce dépôt un poinçon en os poli se trouvait fiché. Découverte évocatrice de pratiques d'accompagnement d'une grande résonnance pour la communauté soucieuse de rétablir au sol, avec exactitude, les signes du statut de cet individu, chargé d'un enfant. Aucune information n'accompagnait l'enfant. 

nouveaux repères stratigraphiques, il a établi une équivalence entre l'ancien niveau 1 et factuelle couche $\mathrm{V}$, d'une part, puis entre l'ancien niveau argileux désigné ici comme III et factuelle couche X. S. Hachi situe dans sa couche IV et sous une anfractuosité de la paroi sud de l'abri ( $2 \mathrm{~m}$ d'ouverture x $1,5 \mathrm{~m}$ de profondeur) huit sujets adultes regroupés. Puis il annonce la découverte de deux autres sujets inhumés contre la paroi dans sa couche $X$. Ses travaux confirment le caractère de nécropole du site et l'existence de sépultures intentionnelles organisées à l'intérieur des mêmes ensembles sédimentaires.

Nous ne disposons pas de datations en relation directe avec les inhumations profondes H 28 et H 16 dégagées en 1927. Les premières datations obtenues sur charbons n'atteignent pas encore la couche X ; pour la couche VII, ces datations s'installent entre 16 577-15 243 cal BC. On peut donc, provisoirement, considérer les deux inhumations profondes comme antérieures à ces repères, et presque contemporaines de celles de Taforalt.

lanfractuosité aucune datation n'a été établie, l'on ne peut pas, pour l'instant, la mettre en relation avec la couche IV, bien qu'elle s'y inscrive, ni avec l'une des datations obtenues sur charbons en trois endroits différents, parfois assez éloignés de l'anfractuosité, et s'échelonnant entre 14 721-12 373 cal BC; $13635-12083$ cal BC; 12 413-11 $557 \mathrm{cal} \mathrm{BC}$.

A propos des huit inhumations de cette anfractuosité, contenues à l'intérieur d'un espace choisi, peu banal, bien circonscrit, les observations signalent :

«Un amoncellement d'ossements des membres inférieurs et supérieurs au niveau de l'ouverture de l'anfractuosité [...]. Tous étaient alignés dans le sens est-ouest, barrant l'ouverture de l'anfractuosité. Puis apparut le crâne de H 1 à l'extrémité ouest avant que ne se découvrent, vers le fond de la niche, six autres crânes (H II-H VII). Le crâne de H VII se tenait à l'extrémité est de l'ouverture. H 1 parut inhumé en décubitus latéral, vraisemblablement fléchi, si l'on en juge par la courbure du rachis, qui parut forcée » (Hachi 2006, p. 433-434).

Les doigts de la main droite, ajoute l'auteur, étaient repliés vers le corps, la même position poing fermé affectait aussi la main gauche. Les six autres sujets présentaient peu d'ossements en connexion anatomique. Seules les inhumations de H III et H VII conservaient vertèbres et côtes en connexion ainsi que H VIII, dont on enregistra le maintien en bonne position du rachis, du bassin et des côtes. S. Hachi les a considérées comme primaires et successives pour ces sept sujets, placés en position fléchie ou contractée, compte tenu de l'exiguïté de l'espace, le huitième pouvant avoir été allongé en avant du groupe comme pour mieux le contenir et obturer la cavité. La position contractée des corps enfoncés dans l'anfractuosité a laissé supposer la mise en place d'un conditionnement individuel particulier, avant la mort de l'individu, déjà recroquevillé n'ayant pas atteint le stade de rigidité cadavérique (corps ligoté et comprimé par des lanières, enveloppé ensuite dans des peaux). Lorsque ces liens organiques disparurent les ossements privés de ligaments se déconnectèrent. Cette vision de plusieurs corps désarticulés qu'eurent les chercheurs (dont C. Arambourg) au moment des fouilles pourrait être à l'origine de la terminologie initiale inadéquate adoptée. On constatera plus loin que cette hypothèse a aussi été retenue pour certaines inhumations de la nécropole atlantique de Skhirat. 

les plus anciennes, antérieures à 16 577-15 $243 \mathrm{cal} \mathrm{BC}$. Elles se situent contre la paroi sud de l'abri sous roche et sont constituées de deux sujets humains $\mathrm{H}$ IX et $\mathrm{H}$ X et du squelette d'un macaque » (Ibid. p. 434). L'individu H IX conserve « un rachis complet en connexion avec ses côtes et son bassin ainsi que l'humérus droit. Ni le crâne, ni les os du membre supérieur gauche, ni les os des membres inférieurs n'ont été trouvés en fouille. Le rachis est allongé dans le sens est-ouest, avec la tête (si elle avait été retrouvée) à l'ouest. Le sujet semble avoir été inhumé en décubitus dorsal, mais rien ne permet de préciser s'il avait été fléchi ou allongé ».

Séparé par une dalle calcaire ( $70 \mathrm{~cm}$ de longueur) plantée de chant,

« le sujet $\mathrm{HX}$ était complet, inhumé sur le côté gauche en décubitus latéral allongé, la tête à l'ouest regardant vers l'ouverture d'abri [...] le bras gauche était allongé avec la main posée dans la région du pubis et le bras droit fléchi avec la main ramenée dans la région du menton et tenant un couteau en os. Le sujet reposait sur de grands éléments de broyage en diabase, portant des traces d'ocre rouge, l'un dans la région du bassin, l'autre dans la région de l'épaule. Le sujet était allongé dans le sens est-ouest. La surface immédiatement attenante au crâne était pavée, sur $1 \mathrm{~m} 2$ de plus de 60 galets et molettes de diverses dimensions et de natures pétrographiques variées : diabase, calcaire siliceux, grès, silex, schiste. Sur cette même surface ont été retrouvés de nombreux éclats en quart-zite issus d'un même nucléus, débité à la pierre. Ces éclats non retouchés, aux arêtes fraîches, ont été débités sur place pour faire partie du dépôt sépulcral, que complètent quelques pierres calcaires issues des chutes des parois et de la voûte de l'abri. La surface était organisée de sorte que les éléments lithiques auxquels se joignent quelques esquilles soient jointifs » (Ibid. p. 434).

La présence du corps complet d'un macaque* n'a pas qu'une incidence biogéographique, on peut établir un lien avec l'enfant et ses jeux avec son animal familier, apprivoisé. Ce nouveau rapport ludique à l'animal serait à rapprocher de celui établi avec la création de figurines zoomorphes à vocation symbolique. Ne pourrait-on pas saisir ces signes de comportements novateurs, comme précurseurs de l'apprivoisement d'autres animaux?

30 Ce qui fait l'originalité de l'abri d'Afalou Bou-Rhummel est certes l'aménagement de sépultures individuelles et multiples dans le respect du statut des inhumés, mais c'est aussi la découverte inattendue des premières créations artistiques de représentations anthropomorphes et archéozoologiques, modelées dans de l'argile, devenues après cuisson de fragiles figurines. S'appuyant sur la distribution ininterrompue des figurines dans les dix couches, S. Hachi souligne l'importance de la transmission des connaissances à certains membres de leur communauté pendant 4000 ans au moins. Même si ces figurines ne semblent pas directement associées aux sépultures, elles livrent divers messages techniques (maîtrise des foyers, de l'argile) et symboliques :

« Nous lions cette production au statut particulier attribué par les groupes humains à ce lieu [...]. Que ce gisement soit le seul, dans l'état actuel des connaissances, à en avoir fourni, signifie qu'il s'agissait là d'une activité réservée, révélant des préoccupations symboliques complexes. Si le savoir faire est acquis, il ne fait pas moins l'objet d'un monopole par le sens. » (Hachi 2006, p. 437).

31 Le caractère si spécial de ces œuvres ne laisserait-il pas «entrevoir comme la concrétisation, la mise en forme de pensées complexes dont le contenu pourrait rendre compte d'attitudes relevant de sphères plus symboliques qu'esthétiques et dont la manifestation requiert des conditions et surtout des lieux particuliers investis d'un 
sens consubstantiel à celui de l'œuvre, aussi à titre provisoire, nous oserons parler d'un art à caractère « hiératique » encore réservé » (Ibid. p. 436-437).

Cette conjonction d'informations interdépendantes colore d'une façon très spéciale le fait funéraire, récurrent et collectif, instauré par les Hommes de Mechta-Afalou, fait novateur, caractéristique de la nécropole nord-africaine dès le début de sa création. Celle-ci occupe l'espace total du site d'Afalou comme à Taforalt et imprègne le massif et ses environs d'une solennité, d'une «sacralité " pourrait-on dire, entretenue par des communautés gardiennes dévouées des rituels et d'une ancestrale mémoire; l'anthropologue qui se plonge avec empathie dans les mystères oubliés d'un passé funéraire resurgi ici - si l'on accepte de l'interroger - n'est donc plus réduit à ne percevoir que les résidus osseux dépouillés et dévitalisés d'inhumations successives. Une sémiotique sous-jacente à l'archéologie et l'anthropologie physique, non perçue, voire marginalisée, est restée incomprise par crainte de découvrir peut être une correspondance entre certains signes présents et passés ; une sémantique appropriée à la nécropole préhistorique devrait pouvoir préciser le concept soudainement apparu constitué, sur la rive méridionale de la Méditerranée, avec l'Homme de Mechta-Afalou. On sait que d'autres individus africains, contemporains, du type de Mechta-Afalou, découverts en Nubie ( $\mathrm{Dj}$. Sahaba) présentent des ressemblances anthropologiques et culturelles, mais il n'a pas été montré qu'ils constituèrent une nécropole (Wendorf 1968).

33 Les observations que S. Hachi soumet aux préhistoriens (Ibid. p. 437-438) vivifient l'Ibéromaurusien et la perception de l'Homme inhumé, en ouvrant de nouvelles pistes de réflexion. L'éclairage qu'il jette sur ces ultimes présences humaines invite l'archéologue à n'être plus seulement comptable de faits quantifiables, à dégager son regard du sol minéralogique, pour appréhender un Etre, le scruter par le biais d'un autre examen plus approprié. De la grotte des Pigeons de Taforalt comme de celle d'Afalou reviennent vers nous, Sapiens actuels, les témoignages d'individus semblables à nous ; leurs sépultures et leurs corps sont ceux d'Etres jadis vivants, disparus, au statut défini perpétué après la mort, à l'intérieur de leur communauté, que nous pouvons approcher et reconnaître. La sépulture, premier contact d'un anthropologue avec un corps inanimé, conserve les traces infimes de soins donnés au corps installé là car «le mort est un être ", "le corps abandonné par la vie va être inhumé avec soin dès l'Ibéromaurusien, dans un lieu de vie, dans l'habitat même ou un peu à l'écart. Il va être intégré au milieu de la vie et au milieu des vivants", "le mort est un être social, symbolique" témoin et guide des survivants. Les offrandes parlent encore d'inhumations privilégiées, du lien entretenu entre vivants et disparus. A Taforalt celles de plusieurs enfants de grands chasseurs de mouflons s'accompagnent du dépôt agencé en étoile de trois massacres de mouflons, elles se trouvent soudainement placées, pour nous aussi, sous la double protection bénéfique des habiles caprinés grimpeurs et des pères-chasseurs. Pour Afalou, le couteau tenu encore en main par l'Homme X allongé ne rappelle-t-il pas l'autorité reconnue au-delà de la mort qui n'abolit pas le souvenir?

La constitution de nécropoles, initiative des Hommes de Mechta-Afalou, se répand en Afrique du Nord vers la fin du Pleistocène et durant l'Holocène. G. Camps signale jusqu'au Néolithique, 29 gisements nord-africains totalisant 500 individus environ (Camps 1974, p. 81). Estimation qui a changé depuis. Après l'Ibéromaurusien, c'est en territoire atlasique à Columnata en particulier que P. Cadenat (1957, 1969 1966, 1968, 
1973-1974) fait connaître la troisième grande nécropole assurant la transition avec d'autres cultures de l'Holocène.

\section{Les nécropoles atlasiques de l'épipaléolithique}

\section{La nécropole de Columnata (Algérie centrale)}

Le gisement des environs de Tiaret (Hauts-Plateaux oranais) a été présenté dans l'EB. L'évocation du site occupant la pente au pied d'un Kef*, en recul, a conduit à souligner la spécificité et l'attractivité des lieux qui abritèrent, presque sans interruption, plusieurs communautés de chasseurs-collecteurs. Une stratigraphie complexe, plus latérale que verticale, inscrit les occupations successives sur une durée d'environ cinq mille ans, de quatre cultures successives : l'Ibéromaurusien (11 sujets), le Columnatien* (36 individus), le Capsien* supérieur (néant) et le Néolithique (2 sujets). M.-C. Chamla $(1970$, p. 18) a estimé à 116 individus le total des sujets dont 48 adultes et 68 enfants et adolescents, tandis que G. Camps n'en n'évoque que 105 (Camps 1974, p. 81, tableau 13).

Vers 10-12 000 BP s'installent des communautés d'Hommes de Mechta-Afalou et une culture ibéromaurusienne modifiée, correspondant à un stade final (fouille 1956, non daté, in Camps p. 79, tableau 11) dans laquelle on découvre les sépultures de 11 individus. Ces inhumations ne conservent rien des anciennes pratiques funéraires. Et nous ne savons pas quand, ni où, les nouvelles apparurent. Aucun individu complet n'a été retrouvé. Les inhumations sont désormais individuelles et secondaires, elles regroupent des ossements épars, sans connexion anatomique qui furent placés dans des structures en pierre. On ne sait rien du stade primaire qui précéda ce fait funéraire. Les premières structures ibéromaurusiennes pourraient ressembler à des tumulus surmontés de rangées de pierres destinées à recevoir des offrandes animales. D'autres architectures plus complexes contenaient les individus H 25, H 26, H 27. La sépulture d'H 25 présentait une structure horizontale: il s'agissait d'une pierre oblongue, fusiforme, déprimée dans la partie centrale posée à plat sur un amas de cendres. A l'intérieur se trouvaient les restes post-crâniens, non calcinés d'un sujet masculin. D'autres éléments humains, d'individus désarticulés, se trouvaient réunis sur un petit espace $(0,40 \mathrm{~m} / 0,50 \mathrm{~m}$ de diamètre et d'épaisseur). Un peu plus loin et en contre bas, s'étendait un pavage $(0,50 \times 1,00 \mathrm{~m})$ de pierres non brûlées et une dalle placée de chant. Cette seconde structure a été désignée comme suggérant un «autel » par P. Cadenat (1957). Avec H 26, P. Cadenat a décrit une troisième structure sub-conique, composée de trois grosses pierres dont deux étaient situées au dessous d'une dalle horizontale. D'autres pierres plus petites étaient éparpillées tout autour d'un individu désarticulé réduit à quelques restes osseux conservés en connexion. Quelques silex taillés furent recueillis dans les sédiments.

Quant au quatrième monument attribué au sujet $\mathrm{H}$ 27, il apparut volumineux et composite, P. Cadenat le laissa sur place. Il mesurait plus d'un mètre de longueur sur $0,50 \mathrm{~m}$ de largeur et 0,30 à $0,40 \mathrm{~m}$ de hauteur. Edifié sur un banc rocheux, ce monument hétérogène, fragile, se composait de petites pierres d'où sortaient les chevilles osseuses et des fragments de bucranes de cinq à six bovidés, posés à plat. La présence de Bos primigenius a été interprétée comme étant une offrande particulière. Certes, mais n'estil pas permis désormais d'interpréter ces témoins fauniques comme des signes révélateurs d'un attachement spécial des hommes de Columnata à cet animal? Ces 
cornages ne seraient-ils pas plutôt non des trophées de chasse, mais des témoins d'un processus d'apprivoisement de l'animal (lait), synchrones des premiers comportements particuliers engagés dans le désert égyptien et en Libye tassilienne (Barich, notice N42) ? Venant après l'exemple d'Afalou (macaque, figurines animales), ce nouvel exemple suggère une nouvelle hypothèse et permet d'entrevoir désormais une mutation comportementale.

Dans le niveau postérieur, culturellement rattaché au Columnatien* (vers $8000 \mathrm{BP}$ ) on constate une nette rupture comportementale, les inhumations sont toutes primaires pour quatre individus (H 8, H. 15, H.21 et H.22) bien individualisés. Elles ont été placées dans des fosses naturelles ou creusées dans le substratum rocheux. Les squelettes sont assez bien conservés. Le sujet féminin $\mathrm{H} 8$ a été déposé en décubitus dorsal fléchi. Cependant la tête, latéralement déplacée s'est détachée du rachis cervical. Son bras droit avait été replié sur la poitrine, le gauche traversait la cage thoracique, les doigts crispés de la main gauche devaient retenir quelque chose de volumineux et résistant, mais périssable, qui a disparu. P. Cadenat très ému par ce dernier geste conservé d'un Etre encore vivant, quoique figé, a suggéré une attitude de souffrance. Cette première confession compatissante de la douleur constitue la plus intime relation d'un fouilleur soudain relié par empathie à la dernière attitude humaine d'un Etre disparu. Image hors du temps présent et que le temps passé n'a pas fait disparaître. Sept petites pierres brûlées ont été retrouvées à l'intérieur du bassin, près de la région lombaire, associées à une pierre plus grande, dressée sur le bassin. Quelques pièces lithiques et osseuses étaient conservées dans les sédiments.

Pour H 15, un monument volumineux formant un socle d'une hauteur d'au moins $0,80 \mathrm{~m}$, avait été dressé, constitué de pierres moyennes empilées sur deux ou trois rangées, de $0,80 \mathrm{~m}$ de diamètre. Au centre devait se dresser un "pilier» parallélépipédique mesurant $0,78 \times 0,20 \times 0,20 \mathrm{~m}$ aux angles émoussés. Rien ne concernait le corps, ou ce qu'il en restait.

L'inhumation du sujet H 21 est celle d'un enfant accroupi, au corps forcé et tassé de manière à s'inscrire dans l'espace étroit d'une fosse naturelle, distante d'un mètre seulement du sujet féminin $\mathrm{H}$ 8. Les épaules se sont trouvées comprimées, la tête s'est introduite dans la cage thoracique, les membres disloqués, le rachis cervical brisé, tout le corps s'est affaissé. Alors que les jambes, fortement écartées, avaient été repliées en arrière. Dans les sédiments plusieurs pièces microlithiques furent retrouvées par tamisage. Or ce sont précisément ces documents si caractéristiques en raison de leur nanisme qui contribuèrent au rattachement de cette sépulture sans contestation possible au faciès Columnatien.

41 Enfin H 22, jeune sujet féminin d'environ 20 ans, a été inhumé sur le même banc rocheux qu'H 8, non creusé mais présentant de larges "sinus " et des sédiments meubles. Le corps posé en décubitus dorsal fléchi présentait le fémur et le genou gauche fléchis et dressés tandis que le droit avait été replié horizontalement, le sacrum reposait sur une pierre. Le corps apparut au fouilleur comme abandonné en position de repos, et fit penser à une mort survenue pendant le sommeil.

On doit à M.-C. Chamla (1970) l'examen de ces individus au squelette gracilisé, ne ressemblant que de façon atténuée au type de Mechta-Afalou et auquel elle donna le nom de Mechtoïdes. 
Après l'exemple de Columnata et le fonctionnement (resté énigmatique) des rituels funéraires de sa nécropole, très différents des cas précédents, il semblerait que plus aucun site du Capsien s.l. n'ait constitué, de la même façon, dans le lieu même d'un habitat choisi, une nécropole, dont les caractéristiques conservent le même sens que celui initialement donné par les Hommes de Mechta Afalou. Pourquoi ? Trois séries de réponses peuvent être apportées.

-Changement climato-comportemental: après le début de l'Holocène et l'installation de conditions climatiques favorables à un habitat dispersé en plein air, les communautés capsiennes éparpillées occupèrent divers territoires, faisant éclater la forte cohésion sociale initiale fondée sur le regroupement humain, typique des sociétés successives de MechtaAfalou dans les massifs côtiers. On peut admettre que l'emprise des hommes sur ces territoires à mouflons permit de maintenir forte et efficace la structuration des groupes de chasseurs. Considérations territoriales et comportementales qui furent perdues avec l'éparpillement des groupuscules capsiens.

- Changement démographique : le nombre des inhumés dans les escargotières capsiennes n'a plus jamais été aussi élevé que dans les grottes de l'Ibéromaurusien. Et $a$ fortiori dans celles de Taforalt et d'Afalou.

- Changement culturel: les Méchtoïdes moins nombreux que les Hommes de Mechta, cohabitant avec d'autres individus Proto-méditerranéens répandus en Afrique du Nord, ont perdu leurs attaches avec un code comportemental ancestral précis qui réglait et organisait les étapes de la vie ibéromaurusienne, code jadis respecté et transmis.

Pourtant, comme G. Camps le rappelle, ces populations du début de l'Holocène :

«Comme les hommes ibéromaurusiens [...] donnaient une sépulture à leurs morts, comme eux ils se paraient le corps, comme eux ils exerçaient sur eux-mêmes des mutilations dentaires mais encore n'hésitaient-ils pas, sans doute dans des buts magiques, à utiliser les ossements humains pour en faire des instruments ou des coupes ou des trophées » (Camps 1974, p. 172).

\section{La nécropole de Medjez II}

Pour rendre compte des pratiques funéraires capsiennes et du concept de nécropole en évolution, évident à Columnata, il aurait été opportun d'évoquer aussi l'édification de l'escargotière de Mechta el-Arbi et le traitement donné aux 22 adultes et aux 14 jeunes enfants qui y furent déposés, mais aucune information ne fut publiée par A. Debruge. Ce sont les anthropologues qui établirent la présence des Hommes de Mechta-Afalou et des Proto-méditerranéens et isolèrent les types anthropologiques que nous utilisons aujourd'hui.

L'escargotière de Médjez* II reste, semble-t-il, la référence la mieux étudiée pour évoquer le fait funéraire Capsien, en évolution pendant les 2500 ans que dure un seul faciès culturel, le Sétifien (Camps-Fabrer 1975 et EB XXXI, p. 4823-4834). Quinze individus y furent reconnus. On doit sans hésitation la considérer comme une nouvelle nécropole d'un type différent en raison de son milieu et de son caractère privatif « il ne s'agissait rien moins que d'une nécropole en escargotière " (Roubet, EB XXXI, p. 4827). On rappellera l'adoption de trois modes d'inhumations primaires, comparables à ceux des Hommes de Mechta-Afalou. La position en décubitus latéral fléchi a été réservée à quatre sujets (H 3, E 1, E 2, E 4), la position forcée entraînant la désarticulation ne fut 
observée qu'une seule fois (H 4), le décubitus dorsal allongé fut adopté pour quatre autres sujets (H 1, H 2, E 3, E 7). Aucune superstructure rocheuse n'avait été édifiée à l'intérieur de la vaste escargotière pour signaler l'emplacement des corps inhumés à différentes hauteurs et en différents emplacements du site. Les travaux ont mis en évidence des sépultures individuelles et d'autres regroupées lorsqu'il s'agissait de jeunes enfants.

Dans une perspective diachronique, c'est l'individu féminin H4, Mechtoïde, qui fut d'abord inhumé alors que l'installation de l'habitat s'était déjà organisée. La position du corps fortement contracté voire désarticulé suggéra l'adoption d'un conditionnement spécial, comparable semble-t-il à celui décrit pour les individus d'Afalou rangés dans l'anfractuosité. Le visage également tourné à l'est. Cet exemple laissa supposer le maintien et le respect d'ancestrales pratiques funéraires installées par les MechtaAfalou.

49 Vinrent ensuite plusieurs individus tous Proto-Méditerranéens. Les nouveaux-nés E 4, E 5, E $6 \mathrm{E} 2$ et $\mathrm{E} 1$, ont connu un traitement très attentionné. E6 reposait sous « un lit d'ocre rouge ", $\mathrm{E} 5$ et $\mathrm{E} 6$ étaient accompagnés de l'offrande d'ossements d'animaux. L'ocre rouge avait été placé près du crâne et des bras d'E 6. Plus haut dans la séquence stratigraphique, à côté de la tête de l'enfant $\mathrm{E} 2$ une coquille marine avait été déposée.

Pour les adultes, l'un féminin $\mathrm{H} 2$, les autres masculins $\mathrm{H} \mathrm{1,} \mathrm{H} \mathrm{3}$, plusieurs offrandes installées sur le corps ou très près de lui, étaient composées d'ossements isolés d'animaux (carnivore, léporidé, mâchoire de canidé), d'un trapèze, et surtout de fragments d'ocre, de parure tubulaire, d'une canine perforée et de poinçons en os poli. Nul dénuement dans l'accompagnement de ces individus de tous âges, au contraire, un soin donné à l'emplacement du corps, à sa position, son orientation, et aux offrandes.

On doit aux notations nombreuses et précises de H. Camps-Fabrer de connaître la nature de l'accompagnement matériel, selon les âges et les individus et de pouvoir percevoir des rituels très organisés. Une gestuelle funéraire conditionnée et appropriée, peut être reconstituée tenant compte du lieu d'inhumation choisi, de la taille, de l'âge et des attaches et traditions culturelles, dans le respect des différences. A Medjez II site du Capsien supérieur, une communauté égalitaire a maintenu des rites et des pratiques voisins de ceux des Mechta-Afalou. Cependant aucun haut personnage n'y a été inhumé, semble-t-il. D'autre part, on n'a pas pu soustraire ces individus inhumés d'un environnement quotidien, sur place et à l'entour. L'intimité, voire la promiscuité des vivants et des morts, fut évidente et permanente comme chez les Mechta-Afalou. On pourrait percevoir à travers le traitement privatif du fait funéraire de ces Capsiens et le contenu entier de leur contexte culturel, le vécu d'une petite société apparemment non hiérarchisé. Ce serait certainement inexact.

\section{Le fait funéraire en nécropole et la néolithisation}

52 L'état actuel des connaissances conduit à reconnaître que la néolithisation s'accompagne en Afrique du Nord d'une documentation très ancienne, souvent non fiable, non exploitable. Les sépultures connues en grottes et sous abris proviennent de recherches hâtives qui privent le chercheur d'une réactualisation.

Pourtant des notations glissées par hasard dans les rapports (celui de Latapie en 1912 après ses fouilles au Damous el-Ahmar, cf. Roubet, N40) permirent de supposer qu'il ne 
se produisit pas de rupture comportementale dans le traitement du fait funéraire entre l'Epipaléolithique final et le Néolithique. Le caractère dispersé de l'habitat situé en altitude, sous abri et en grotte, en région atlasique, conduisit à maintenir le caractère privatif, de la nécropole, réservée semble-t-il à une petite communauté pastorale. C'est le cas dans les Némencha, du site du Damous el-Ahmar. Les quatre inhumations découvertes dans un couloir adjacent à l'Abri $\mathrm{n}^{\circ} 2$, étaient primaires, parfois installées dans des cistes (coffres), l'adulte $\mathrm{H} 4$ en décubitus latéral fléchi. La tête de l'individu $\mathrm{H}$ 3 , avait été enduite d'ocre rouge.

Pour le Néolithique tellien (Roubet et Kherbouche, N46) c'est-à-dire méditerranéen (Camps 1974, p. 262-280), les travaux anciens dans le massif du Murdjadjo, en Oranie littorale signalèrent dans la grotte de l'Aïn Guettara, notamment des inhumations dont la dernière dégagée en 1967, fut celle d'un individu adulte du type de Mechta-Afalou, sans agencement ni offrandes, retrouvé vers le milieu de l'habitat, dans le niveau III. Rien ne permet d'envisager, pour le moment, le fonctionnement du lieu autrement que privatif.

Que savons-nous du Maroc postérieurement à Taforalt? Deux nécropoles ont été retenues, l'une à El-Kiffen l'autre à Skhirat qui fait l'objet de récents travaux.

\section{La nécropole du littoral Atlantique d'El-Kiffen}

Dans la région de Casablanca, le site des falaises marines d'El-Kiffen abrita une nécropole néolithique, ayant contenu 17 individus au moins (Bailloud et Mieg de Boofzheim, avec la collab. de Balfet et Kiefer, 1964). Le compte rendu des fouilles de 1956, 1961 et 1962 précise sur plans les nombreux emplacements des individus identifiés entre les derniers dépôts du site (sous $0,60 \mathrm{~m}$ de profondeur) et le niveau profond, naturel et humique, archéologiquement stérile, apparu après $1,40 \mathrm{~m}$.

Cette première étude s'accompagne de tant de données associées et très minutieuses, qu'il semble délicat d'opérer un cloisonnement entre les faits anthropiques, les rituels, et les dépôts à l'intérieur desquels ils s'inscrivent. Ces dépôts sont tous antérieurs aux inhumations, ce seraient ceux d'un atelier plus ancien, épipaléolithique certainement (Ibid. p. 166), s'étendant hors de la zone occupée par les inhumations «dont les matériaux ont été obligatoirement, au cours des opérations de creusement et de remblaiement des fosses sépulcrales, mêlés secondairement au mobilier accompagnant les sépultures" (Id. p. 160). Toutes les inhumations se sont trouvées associées à des nombreux récipients céramiques: 43 vases qu'on put reconstituer (études technologiques de H. Balfet, p. 138-143 et de Ch. Kiefer, p. 144-153). Par leur qualité, leurs caractères typiques, leur lien au contexte funéraire, ces vases sans contenu d'incinération contribuent à donner à cette nécropole un aspect original, une dimension comportementale, sociétale, sans équivalent.

Les données radiométriques trop anciennes, faites à Auckland (Nouvelle Zélande) en 1958, s'accompagnent d'un commentaire dubitatif, exprimé par le laboratoire ; en réalité elles ne seraient plus fiables (Cf. p.168-169). Elles résultent d'échantillons d'ossements humains datés entre $4300 \pm 80$ BP (soit 2342 av. J.C.) pour l'une, et $3100 \pm 200$ BP (soit 1142 av. J.C) pour l'autre. On peut rappeler qu'il s'agit de contextes très postérieurs au Cardial (Ballouche et al., N47).

59 Les 17 inhumations sont représentées par 15 crânes, confiés en France au Dr. Riquet. Leur étude anthropologique s'avéra délicate compte tenu de l'état des documents. 
Même si le type méditerranéen a été initialement reconnu «les inhumés d'El-Kiffen paraissaient appartenir, dans leur ensemble, à la race méditerranéenne » (Bailloud et al. p.113) il ne semble pas que d'autres résultats l'aient confirmé. Qu'apportent les informations évoquant « le rituel funéraire » (Ibid. p. 103-113) ? Elles se résument ainsi : dans les emplacements bien localisés sur plan ces inhumations sont collectives, parfois même simultanées et non successives (ibid. p. 108), sans aménagement de sépultures, la plupart sont secondaires, quant aux offrandes, réduites à quelques vases par individu, il s'agirait «non de pièces neuves, mais d'objets usagés, ou détériorés souvent incomplets" (Ibid. p. 103). La sépulture primaire du squelette VII, en E3-4, fait exception (Ibid. p.106), l'individu gisait au fond d'une fosse, en décubitus dorsal, bras fléchis, les mains reposant sur le ventre, les jambes légèrement fléchies, pieds joints. Aucune autre notation n'évoque d'individu complet, ni au statut reconnu. Le vocabulaire adopté pour évoquer les inhumations collectives, secondaires, anatomiquement si déconcertantes, reste parfois inadéquat. Cette nécropole exceptionnelle, ici indépendante de tout habitat contemporain (Ibid. p. 113) mérite une réactualisation de la documentation. S'il s'avérait établi que les datations sont si récentes (âge du Bronze), alors ce site, aux influences ibériques et almériennes nombreuses, et au maintien de certaines traditions lithiques préhistoriques nordafricaines, donnerait un exemple d'africanisation des modèles comportementaux le long de l'Atlantique.

\section{La nécropole de Skhirat} plein air, dans une sablière, au sein d'un cordon dunaire, près d'un estuaire. Entre 1982 et 1984, au cours de trois campagnes de fouilles (J.-P. Daugas et F.-Z. Sbihi-Alaoui) une centaine d'individus et 14 dépôts funéraires ( 87 sépultures et 11 crânes, collectés hors stratigraphie avant les fouilles) furent mis au jour, dans une couche estimée contemporaine et postérieure d'un kjoekkenmödding (Lacombe 2001, 2004). entre 4380 et 4300 avant notre ère.

«La nécropole de Skhirat apparaît originale, non seulement de par sa situation géographique, mais aussi de par sa structure intrinsèque avec une répartition démographique comportant de nombreux immatures... représentés par des périnataux et des nourrissons » (Lacombe, p. 156).

Divers objets accompagnent ces enfants, il s'agit de haches polies, de perles en coquille d'œuf d'autruche, de pendentifs en coquillage, de bracelets et d'ocre rouge. Outre la mortalité infantile, élevée ici aussi, il semble que le fait funéraire soit indicatif d'une population « vivante » originelle (Lacombe 2004, p. 157). Plusieurs modes d'inhumation des immatures sont représentés, en décubitus latéral, souvent droit ou en position très contractée " évoquant un transport du cadavre... » dans « ... un contenant en matériau périssable servant de linceul » (Ibid. p. 157). D'autre part, les observations sur le terrain ont conduit à remarquer une organisation de l'espace sépulcral «regroupant 2 à 3 individus, enfants ou adultes appartenant à une même famille qu'elle soit " génétique ", « sociale » ou tout simplement " affective ", mais dans l'ensemble il s'agit de sépultures uniques et isolées" (Ibid. p. 157); dans certains cas, une association intentionnelle de deux sépultures a été déduite de la position symétrique des corps inhumés face à face. Divers travaux multidisciplinaires sont en cours.

Encyclopédie berbère, 33 | 2012 
63 Ayant passé en revue, en région septentrionale les données majeures du fait funéraire conservé dans les principales nécropoles préhistoriques, on constatera que c'est précisément vers le milieu et la fin de l'Holocène qu'il aurait été utile de rassembler plus d'informations régionales. Rien d'assez précis n'autorise à fixer le cadre conceptuel, comportemental et métaphysique des populations installées avant l'apparition et la généralisation du mégalithisme. Un hiatus chrono-culturel occupe cette position charnière: aucun signe précurseur, excepté certaines sépultures construites de Columnata, n'a été découvert pour annoncer les motivations d'une insertion sur les collines et les crêtes des paysages, du fait dolménique qui signe la séparation nette des vivants et des morts, caractéristique de l'entrée dans une période protohistorique, qui vit surgir en toutes régions de très nombreuses nécropoles.

\section{BIBLIOGRAPHIE}

ARAMBOURD C., BOULE M., VAlLoIS H.-V., VERnAU R., 1934 - Les grottes paléolithiques des Beni Segoual (Algérie), Paris, Masson (Archives de L'Institut de Paléontologie Humaine, $\mathrm{n}^{\circ} 13$ ).

BAILLOUd G., MIEG DE BOOFZHEIM, BALFET H., KIEFER Ch. 1964 - « La nécropole néolithique d'El Kiffen, près des Tamaris (Province de Casablanca), Maroc », Libyca, XII, p. 95-191.

BARBIN A., 1912 - «Fouilles des abris préhistoriques de la Mouillah près de Marnia (deuxième campagne) », Bull. de la Soc. de Géographie et d'Archéologie de la province d'Oran, t. XXXII, p. 389-402.

BARTON R.N.E., BOUZOUGGAR A., BRONK-RAMSEY C., COLLCUTT S.N., HIGHMANN T.F.G., HUMPHREY L., PARFITT S., RHODES E.J., SCHWEINNIGER J.-L., STRINGER C., TURNER E., WARD S., 2007 - “Abrupt climatic change and chronology of the upper Palaeolithic in northern and eastern Morocco", in : Mellars P., Boyle, K., Bar-Yosef O., Stringer C. (Eds.), Rethinking the Human Revolution : New Behavioural \& Biological Perspectives on the Origins and Dispersal of Modern Humans. Research Monographs of the Macdonald Institute, Cambridge, p. 177-186.

BOUZOUGGAR A., BARTON R.N.E., BLOCKEY S., BRONK-RAMSEY C, COLLCUTT S.N., GALE R., HIGHMANN T.F.G., HUMPHREY L.T., PARFITT S., TURNER E., WARD S., 2008 - "Reevaluating the age of the Iberomaurusian in Morocco", African Archaeological Review 25 (1 et 2), p. 3-19.

CADENAT P., 1957 - « Fouilles à Columnata 1956-1957. Première partie ; la nécropole », Libyca, t. V, p. 49-81.

CADENAT P., 1959 - « Principaux résultats des fouilles exécutées en 1956-1957 à Columnata », Congrès Préhistorique de France, 16e session, Monaco, p. 316-320.

CADENAT P., 1966 - « Vues nouvelles sur les industries de Columnata », Libyca, t. XIV, p. 189-207.

CAMPS G., 1974 - Les civilisations préhistoriques de l'Afrique du Nord et du Sahara, Paris, Doin.

CAMPS-FABRER H., 1975 - Un gisement Capsien de faciès Sétifien : Medjez II. El Eulma (Algérie). Etudes d'Antiquités Africaines. Avec la collab. de J. Bouchud, M. Chabeuf, M.-C. Chamla, M. Couvert, R. Dughi, et F. Sirugue. CNRS, 448 p. CHAMLA M.-C., DASTUGUE J. et HACHI S., 1985 - « A73. Afalou BouRhummel », Encyclopédie berbère, fasc. II, Edisud, p. 182-193. 
CHAMLA M.-C., 1970 - Les hommes épipaléolithiques de Columnata (Algérie occidentale). Etude anthropologique, Paris, AMG (Mém. XV du CRAPE), 132 p.

COURTY M.-A., VALLVERDU J., 2001 - "The microstratigraphic record of abrupt climate change in cave sediments of the Western Mediterranean", Geoarchaeology (5), p. 467-499.

COURTY M.-A., GOLDBERG P., MACPHAIL R.I., 1989 - Soils and Micromorphology in Archaeology, Cambridge University Press, Cambridge, $344 \mathrm{p}$.

DASTUGUe J., 1962 - « Pathologie des Hommes de Taforalt », in D. Férembach, La nécropole épipaléolithique de Taforalt (Maroc oriental). Etude des squelettes humains, Casablanca, p. 135-158.

DASTUGUE J., 1959 - « Un orifice crânien préhistorique », Bull. et Mém. de la Soc. d'Anthropologie de Paris, t. 10, Xe série, $\mathrm{n}^{\circ} 4$, p. 357-363.

DASTUGUE J., 1975 - «Pathologie des hommes préhistoriques d'Afalou BouBou-Rhummel», L'Anthropologie, t. 79, p. 483-506.

DEBRUGe A., 1914 - « Présentation d'ossements de Mechta-el-Arbi et de la grotte d'Ali Bacha (Constantine) », Bull. de la Soc. Préhist. Française, t. XI, p. 260-261.

DEBRUGE A. et MERCIER G., 1913 - «Présentation d'un crâne et de l'industrie de l'escargotière de Mechta-el-Arbi », Bull. de la Soc. Préhist. Française, t. X, p. 534-540.

FEREMBACH D. 1970 - « Les Cro-Magnoïdes de l'Afrique du Nord », in L'Homme de Cro-magnon, Anthropologie et archéologie sous la dir. de G. Camps et G. Olivier, Paris, AMG, p. 81-92.

FEREMBACH D., DASTUGUE J. POITRAT-TARGOWLA M.-J., 1962 - La nécropole épipaléolithique de Taforalt (Maroc oriental). Etude des squelettes humains, Rabat, ed. Casablanca, $176 \mathrm{p}$.

HACHI S., 1995 - « Résultats des fouilles récentes d'Afalou Bou-Rhummel (Bédjaïa, Algérie) », in J.M. Fullola, N. Soler (Eds.), El Mon Mediterrani desprès del Pleniglacial (18 000-12 000 BP.), Girona, Espagne, p. 77-92.

HACHI S., 1996 - «L'Ibéromaurusien : découvertes des fouilles d'Afalou », L'Anthropologie, t. 100, p. 55-76.

HACHI S., 1999 - L'homme de Mechta-Afalou, Cro-Magnon de l'Afrique de l'Afrique du Nord, durant les 20 derniers millénaires : évolution culturelle et devenir, Thèse d'Habilitation à diriger des recherches, Université Paul Valéry III, Montpellier, 4 volumes.

HACHI S., 2003a - Les cultures de l'Homme de Mechta-Afalou, Alger, CNRPAH (Mémoire CNRPAH, n.s., $\left.\mathrm{n}^{\circ} 2\right)$.

HACHI S., 2003b - Aux origines des Arts Premiers en Afrique du Nord. Les figurines et les objets modelés en terre cuite de l'Abri sous-roche préhistorique d'Afalou, Babors, Algérie. (18 000-11 000 BP). (Mémoire CNRPAH, n.s. $n^{\circ}$ 6), Alger, CNRPAH.

HACHI S., 2006 - « Du comportement symbolique des derniers chasseurs MechtaAfalou d'Afrique du Nord », C.R. Palevol, t. 5, fasc. 1-2, p. 429-440.

HACHI S., FRÖHLICH F., GENDRON-BADOU A., DE LUMLEY H., ROUBET C., ABDESSADOK S., 2002 - « Figurines du Paléolithique supérieur en matière minérale plastique cuite d'Afalou Bou-Rhummel (Babors, Algérie). Premières analyses par spectroscopie d'absorption infrarouge ", L'Anthropologie, t. 196, p. 57-97.

LACOMBE J.-P., 2004 - « Anthropologie du Néolithique marocain. La nécropole de Skhirat : approche chrono-géographique des dysplasies pariétales », Anthropo.7, p. 155-162. 
LACOMBE J.-P. et DAUGAS J.-P., 1988 - « La nécropole néolithique de RouaziSkhirat », Bull. et Mém. de la Soc. d'Anthropologie de Paris, t. V, 16e série, p. 308-309.

ROCHE J., 1953 - « La grotte de Taforalt », L’Anthropologie, t. 57, p. 375-380.

ROCHE J., 1963 - L'Epipaléolithique marocain, Lisbonne, 2 vol.

ROUBET C. et HACHI S., 2000 - « Les sépultures de Mechta-Afalou, Cro-Magnon de l'Afrique du Nord : approche culturelle à partir de nouvelles découvertes », in Ph Andrieux, D. Hadjouis et A.

Dambricourt-Malassé, l'Identité humaine en question. Nouvelles problématiques et nouvelles technologie en Paléontologie humaine et en Paléoanthropolgie biologique, Coll. du Val de Marne 26-28 mai 1999, p. 425-441.

TAYLOR V.K., BARTON R.N.E., BELl M., BOUZOUGGAR A., COLLCUTT S., BLACK S., HOGUE J.T. 2011 - “The Epipalaeolithic (Iberomaurusian) at Grotte des Pigeons (Taforalt), Morocco : A preliminary study of the land Mollusca", Quaternary International, p. 1-10.

WENDORF F. 1968 - The Prehistory of Nubia, Dallas, Southern Methodist University Press, 2vol.. WODWARD J.-C., GOLDBERG P., 2001 - "The sedimentary records in Mediterranean rockshelters and caves : archives of environmental change", Geoarchaeology 16, (4), p. 327-354.

INDEX

Mots-clés : Mort, Préhistoire, Rite 